\title{
USANDO OBJETOS DE APRENDIZAJE EN ENSEÑANZA SECUNDARIA OBLIGATORIA
}

\author{
Isabel Gutiérrez Porlán \\ isabelgp@um.es \\ Grupo de Investigación de Tecnología Educativa \\ Universidad de Murcia
}

\section{RESUMEN:}

En este artículo se presentan los resultados y las principales conclusiones de una experiencia de implementación de Objetos de Aprendizaje para la enseñanza de las matemáticas en 20 de la ESO de un IES de la Región de Murcia. En esta experiencia los profesores del departamento de matemáticas diseñaron, crearon e implementaron los Objetos de Aprendizaje para el trabajo de los alumnos como complemento a las clases presenciales.

\section{PALABRAS CLAVE:}

Objetos de Aprendizaje, OER, matemáticas, Enseñanza Secundaria Obligatoria.

\section{ABSTRACT:}

This article presents the results and main findings of an experimental implementation of Learning Objects for teaching mathematics in a High School in the region of Murcia (Spain). In this experience the teachers of the Department of Mathematics designed, created and implemented the learning objects as a complement to the classroom.

\section{KEY WORDS:}

Learning Objects, OER, Mathematics, Secondary Education. 


\section{DE LOS OBJETOS DE APRENDIZAJE A LOS OER}

Abordar la definición del concepto de Objeto de Aprendizaje es una tarea complicada si tenemos en cuenta la gran cantidad de definiciones surgidas en torno al término y más aun si contamos con el hecho de que desde sus orígenes este concepto ha ido evolucionando y adaptándose a las nuevas necesidades y requerimientos tanto tecnológicos como pedagógicos.

A pesar de que fue en 1994 cuando Daivid Hodgins habló por primera vez de Objetos de Aprendizaje, remarcando que cualquier material digital podía ser diseñado y producido para ser empleado en diferentes situaciones pedagógicas (Hodgins 2000), la idea de que los contenidos digitales podían ser puestos a disposición de todas las personas interesadas en su utilización es bastante más antigua. En 1971, nos encontramos con la iniciativa llevada a cabo por el Proyecto Gutemberg, desarrollado por Michael Hart, cuya finalidad era la creación de una biblioteca de libros electrónicos que ya existiesen físicamente. En la actualidad este proyecto cuenta con más de 20.000 ejemplares a su disposición. Una década después, el 1 de Junio de 1982, se puso en marcha una modalidad de distribución de Software denominada Shareware. La idea era que los materiales fueran distribuidos para ir siendo evaluados y mejorados, a pesar de que al final si se quería adquirir la licencia se hacía necesario realizar un pago (en ocasiones muy modesto) al autor.

Además de las iniciativas anteriores, son muchas más las acciones que han ido surgiendo a lo largo del tiempo en cuanto al trabajo y colaboración con materiales digitales reutilizables. Pero ¿a qué nos referimos exactamente cuando hablamos del concepto de objeto de aprendizaje? Como ya decíamos anteriormente, resulta bastante complicado encontrar una única definición válida y que esté aceptada universalmente ya que existe una amplia discusión respecto del término. ${ }^{1}$

Muirhead y Haughey, (2003), sugieren que las diversas definiciones en torno al término dan un especial énfasis a alguno de los componentes del tándem Objeto de Aprendizaje, unas centrándose más en el hecho de que son objetos y otras en el aspecto educativo.

Si nos centramos en el hecho de que son objetos, encontramos definiciones como la realizada por la NLII (National Learning Infrastructure Initiative, 2003) en Estados Unidos, que se refiere a los objetos de aprendizaje como "recursos digitales siempre modulares que son usados para apoyar el aprendizaje"; o la realizada por el Comité de Estándares de Tecnologías del Aprendizaje, que nos ofrece una definición que resulta ser algo ambigua, dado que se refiere a los OA como "cualquier entidad, digital o no digital, la cual puede ser usada, re-usada o referenciada durante el aprendizaje apoyado por tecnología"(2003). Estas definiciones nos sirven como primera aproximación pero se hace necesario incidir más en el aspecto pedagógico de los OA.

\footnotetext{
${ }^{1}$ Sobre este particular recomendamos la lectura del capítulo Connecting learning objects to instructional design theory: A definition, a metaphor, and a taxonomy de Daivid Wiley (Wiley, 2000), en donde el autor hace un recorrido de todas las definiciones existentes hasta el momento de publicación.
} 
Wiley (2000) asegura que un objeto de aprendizaje puede ser "cualquier recurso digital que puede ser reutilizado para facilitar el aprendizaje". Dentro de esta definición, siguiendo al mismo autor podemos incluir cualquier cosa que pueda ser distribuida a través de la red, sea grande o pequeña. Podemos entender por tanto como recurso digital reutilizable tanto imágenes, fotos, texto, cortos de video o audio, páginas web, presentaciones visuales...

En este punto, el relativo a incidir más en el componente pedagógico, nos parece interesante la definición dada por Varas (2003), ya que consideramos que resume adecuadamente el sentido de los objetos de aprendizaje:

"Los Objetos de Aprendizaje son piezas individuales autocontenidas y reutilizables de contenido que sirven a fines instruccionales. Los Objetos de Aprendizaje deben estar albergados y organizados en Meta-data de manera tal que el usuario pueda identificarlos, localizarlos y utilizarlos para propósitos educacionales en ambientes basados en Web. Los potenciales componentes de un Objeto de Aprendizaje son:

- Objetivo instruccional

- Contenido

- Actividad de estrategia de aprendizaje

- Evaluación"

De la misma manera L'Allier (1997) entiende los Objetos de Aprendizaje como la estructura mínima independiente que contiene un objetivo, una actividad de aprendizaje y un mecanismo de evaluación.

En ocasiones para facilitar su explicación muchos autores han recurrido al uso de metáforas. Wiley (2000) ya utilizó una que posteriormente ha sido empleada en infinidad de explicaciones sobre el tema, "la metáfora del Lego". De todos es conocido el juego de Lego, en el que a base de pequeñas piezas que se pueden reutilizar cuantas veces se desee, se puede construir un castillo, un barco, una nave espacial...por lo que dado un conjunto de piezas, las combinaciones posibles son casi infinitas. Esta es una de las formas más simples que se ha utilizado para explicar el uso pedagógico de los objetos de aprendizaje.

Una alternativa a la metáfora del Lego, también empleada por Wiley, es la de la comparación de los Objetos de Aprendizaje con átomos, ya que para éstos solo existen una serie de combinaciones prefijadas por su propia estructura. Los átomos (y los $O A$ ) requieren a alguien con conocimientos de teoría atómica (instruccional, en el caso de los OA) para combinarlos con resultados satisfactorios. Por tanto es necesario que la estructura de los mismos esté bien definida y explicada en los metadatos que todo Objeto de Aprendizaje contiene. Esta nueva metáfora incide en dos aspectos clave, por un lado la necesidad de que los objetos de 
Aprendizaje contengan información sobre sí mismos y por otro lado la necesidad de que los profesores dispongan de conocimientos y formación respecto al tema.

La última metáfora también empleada por Wiley (2008), es la que compara los OA con un collar de perlas. Las perlas son bellas por sí solas pero adquieren un especial sentido cuando están engarzadas formando un collar. Además de que la forma de este collar puede variar considerablemente dependiendo del tipo de perlas o abalorios que empleemos para formarlo.

Algunas de las definiciones que hemos aportado hasta el momento, hacen referencia al uso de OA como apoyo al proceso de e-a, aunque es necesario matizar que existen otras definiciones que entienden los $\mathrm{OA}$ como entidades que por sí solas pueden facilitar el aprendizaje, como ocurriría en el caso de la teleenseñanza. De estas definiciones que apoyan el uso de OA como únicos recursos posibles para facilitar el aprendizaje, hemos seleccionado la que aportan Relan y Gilliani (1997) que asocian el concepto de Objeto de Aprendizaje con el de formación mediante Internet, y la definen como "la aplicación de un repertorio de estrategias instruccionales orientadas cognitivamente y llevadas a cabo en un ambiente de aprendizaje constructivista y colaborativo, utilizando los atributos y recursos de Internet".

Se hace francamente difícil decantarse por una única definición. No obstante, podríamos resumir el concepto diciendo que:

- Es un material en red.

- Es concebido como herramienta de enseñanza y con un modelo didáctico subyacente y

- Que incorpora un fichero de metadatos que lo describen.

Dicho en pocas palabras y de forma simple, un contenido didáctico en red descrito por unas etiquetas -unos metadatos- constituye un objeto de aprendizaje.

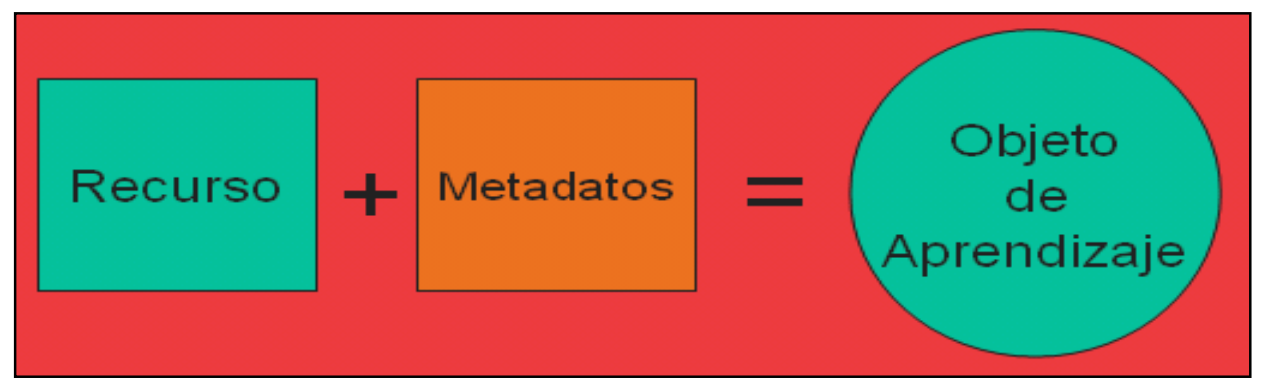

Composición básica de un Objeto de Aprendizaje 
Desde nuestro punto de vista los Objetos de Aprendizaje son la denominación más extendida (e internacional) que designa un medio didáctico reutilizable en red. A continuación matizaremos un poco en las particularidades que esta definición conlleva. Al decir que los OA son un medio, nos referimos a que es un todo complejo que tiene una entidad instrumental (como documento electrónico, como archivo), y una entidad simbólica (que incluye una información, con una estructuración y un lenguaje específico) (Cabero, 1999). Pero los OA no son medios simplemente, sino que son medios didácticos (no de enseñanza), entendido como un elemento (es decir, como objeto en sí mismo) que ha sido diseñado para servir en un proceso educativo (Prendes, 1998) y que además son reutilizables, porque han sido configurados (instrumental y simbólicamente) para poder ser de utilidad en diferentes procesos educativos por usuarios diversos. Los contenidos de aprendizaje se dividen en pequeñas unidades de instrucción apropiadas para poder utilizarlas en varios cursos (Rebollo, 2004:10). Además de lo anterior, los OA están en red, evidentemente en el sentido más tecnológico de la palabra (refiriéndonos a redes telemáticas, interactividad instrumental), pero también en el sentido de redes de profesionales, de aprendices, docentes, etc. (redes sociales, interactividad cognitiva) (Prendes, 1995).

Algunas de las características más importantes que podemos destacar de los mismos son las siguientes (Martínez y Prendes, 2007)

- Breves: Esta característica hace alusión al tamaño de los OA, cuanto más pequeños son, más sencilla será su aplicación en contextos diferentes.

- Independientes: Relacionado con la característica anterior los OA, han de ser independientes, es decir, por si solos han de facilitar el aprendizaje.

- Combinables: los OA posibilitan su agregación con otros OA (recordar las metáforas comentadas anteriormente), aunque el mismo OA puede crear diferentes combinaciones.

Las características comentadas anteriormente hacen que los objetos de aprendizaje nos den la posibilidad de (Rebollo, 2004:10):

- Interoperabilidad: las unidades instruccionales pueden integrarse independientemente de su desarrollador o de la plataforma para la que hayan sido diseñadas.

- Durabilidad: las unidades de instrucción siguen siendo utilizables aunque cambien las tecnologías para su presentación y distribución.

- Accesibilidad: el contenido está disponible en cualquier parte y en todo momento.

Como hemos podido comprobar, desde sus orígenes, el concepto de Objeto de Aprendizaje ha ido evolucionando y cambiando dando lugar a diferentes definiciones e ideas acerca de los 
mismos. A lo largo de esta evolución, ha pasado por momentos polémicos sobre su utilidad, validez y necesidad para la educación ya que no olvidemos que Wiley (2006), principal precursor de este concepto, anunció la muerte de los Objetos de Aprendizaje alegando la poca aplicación que tenían para la educación por la cantidad de requerimientos tecnológicos de los mismos.

Superando todas estas adversidades, nos encontrándonos en los últimos tiempos con un nuevo término, "Open Educational Resource" (OER), utilizado por primera vez en Julio de 2002 durante un Workshop de la UNESCO. Este término hace alusión a recursos educativos (lecciones, glosarios, módulos de instrucción, simulaciones...) que están disponibles para su uso libre, siendo posible rehusarlos, adaptarlos y compartirlos. Otras definiciones del término, incluyen contenidos, software, herramientas y experiencias de buenas prácticas. Los OER están siempre disponibles de manera libre para ser utilizados por cualquier persona que está interesada y suponen una evolución respecto a los OA por esta mayor libertad y amplitud en cuanto a su uso.

\section{LOS OBJETOS DE APRENDIZAJE PARA LA ENSEÑANZA DE LAS MATEMÁTICAS.}

La elaboración de material digital para la enseñanza es una de las tareas que más esfuerzo supone a la mayoría de profesores cuando deciden introducir las tecnologías dentro del aula. Por otra parte el trabajo de los alumnos con estos materiales hace que en muchas asignaturas (incluidas las matemáticas) se puedan presentar los contenidos de una manera más amigable y cercana al alumno y a sus formas de aprender y de codificar la información. Además de que cuando los materiales están puestos en red el alumno cuenta con una mayor libertad y autonomía para utilizarlos y trabajar con ellos en función de sus necesidades.

Elaborar dichos materiales puede suponer una carga de trabajo extra que la mayoría de docentes no pueden asumir de manera aislada y su alternativa clara pasaría por el trabajo profesional en equipo.

Nuestro trabajo se centra en el diseño de material digital en red para la enseñanza de las matemáticas en la ESO, elaborado en equipo y que además pueda ser reutilizable de modo que se facilite el trabajo posterior por parte de otros profesores. Este trabajo se realizó en el IES "La Flota", de la Región de Murcia y concretamente en el departamento de matemáticas de dicho centro, donde actuó de coordinador el profesor José Hernández Franco, director del departamento. En la experiencia participaron los profesores del departamento y los cinco cursos de alumnos de 2o de la ESO.

La experiencia llevada a cabo se diseñó en torno a dos fases generales, una primera fase de trabajo con los profesores y una segunda fase de trabajo con los alumnos. En la Fase de Preparación los profesores diseñaron los OA que se iban a utilizar en el desarrollo de la

experiencia con los alumnos (fase final). El hecho de que éstos crearan sus propios materiales se debe a que a pesar de buscar material ya existente para reutilizar (no olvidemos que la 
reutilización es una de las bases que fundamenta el trabajo con OA), el tema que se acordó para trabajar con $\mathrm{OA}$, respondía a unas características y objetivos muy concretos, por lo cual se optó por diseñar y producir materiales nuevos ajustados a la experiencia específica a realizar y que respondiesen a unos criterios comunes consensuados por el grupo de profesores.

Las herramientas empleadas por los profesores en el desarrollo de la experiencia fueron las siguientes: para el diseño de materiales se utilizó Lectora Profesional Publisher, para generar el repositorio de materiales se utilizó DSpace que es una herramienta de código abierto que permite empaquetar los materiales y generar el almacén y por último para el trabajo con los alumnos se implemento $n$ curso a través de Moodle.

Todos los profesores recibieron un curso previo de formación sobre las herramientas utilizadas, al igual que los alumnos a los que se les dio un curso para el uso y el trabajo en Moodle.

Para la recogida de información de la experiencia se emplearon una serie de cuestionarios (inicial y final) a alumnos y profesores. En estos cuestionaros se preguntaba acerca del uso del ordenador y de Internet (inicial) y sobre el desarrollo de la experiencia de trabajo con OA (final). Los principales resultados obtenidos los presentamos a continuación:

Con respecto a la frecuencia del uso del ordenador e Internet por parte de los alumnos, más de la mitad (69\%) lo hace con frecuencia. Respecto a los profesores todos afirman usar el ordenador e Internet bastante o mucho (algo que resulta bastante predecible si tenemos en cuenta que son profesores que han decidido participar voluntariamente en una experiencia de uso de TIC). La mayoría de los alumnos accede a Internet para actividades relacionadas con el ocio, la comunicación y la descarga de música. Con respecto a la frecuencia en el uso de herramientas ofimáticas por parte de éstos, podemos comprobar en el siguiente gráfico que aquellas más utilizadas son las relacionadas con los servicios de Internet (navegadores, buscadores, chat y mensajería instantánea). La mayoría de los alumnos (88\%), afirma utilizar el ordenador para tareas escolares, sobre todo en las asignaturas de matemáticas, francés y tecnología. 
Frecuencia de uso de herramientas por parte de los alumnos

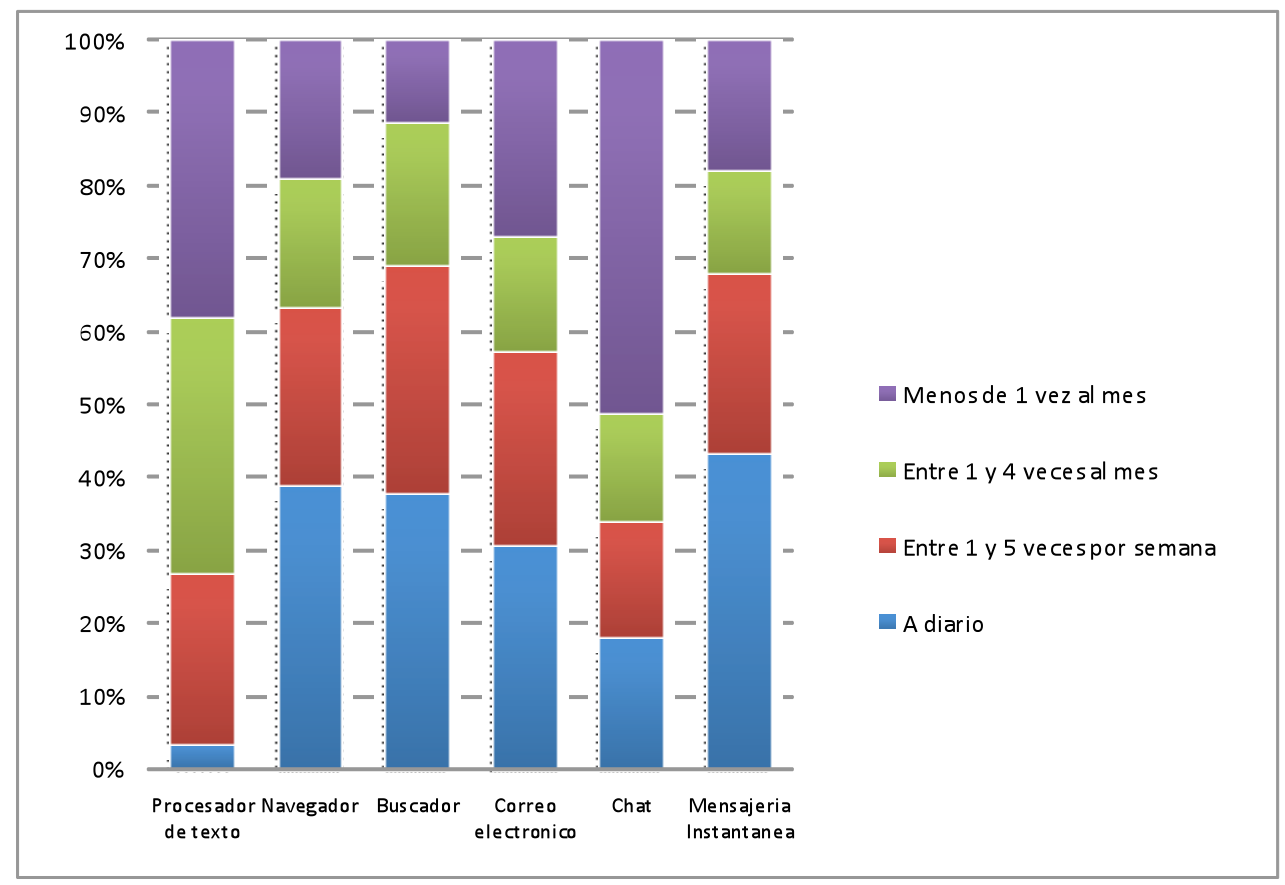

Por otra parte, las herramientas más utilizadas por los profesores son: navegadores y buscadores, chat, correo electrónico y a diferencia de los alumnos se utiliza con más frecuencia el procesador de texto, tal y como podemos comprobar en el siguiente gráfico.

Frecuencia de uso de herramientas por parte de los profesores

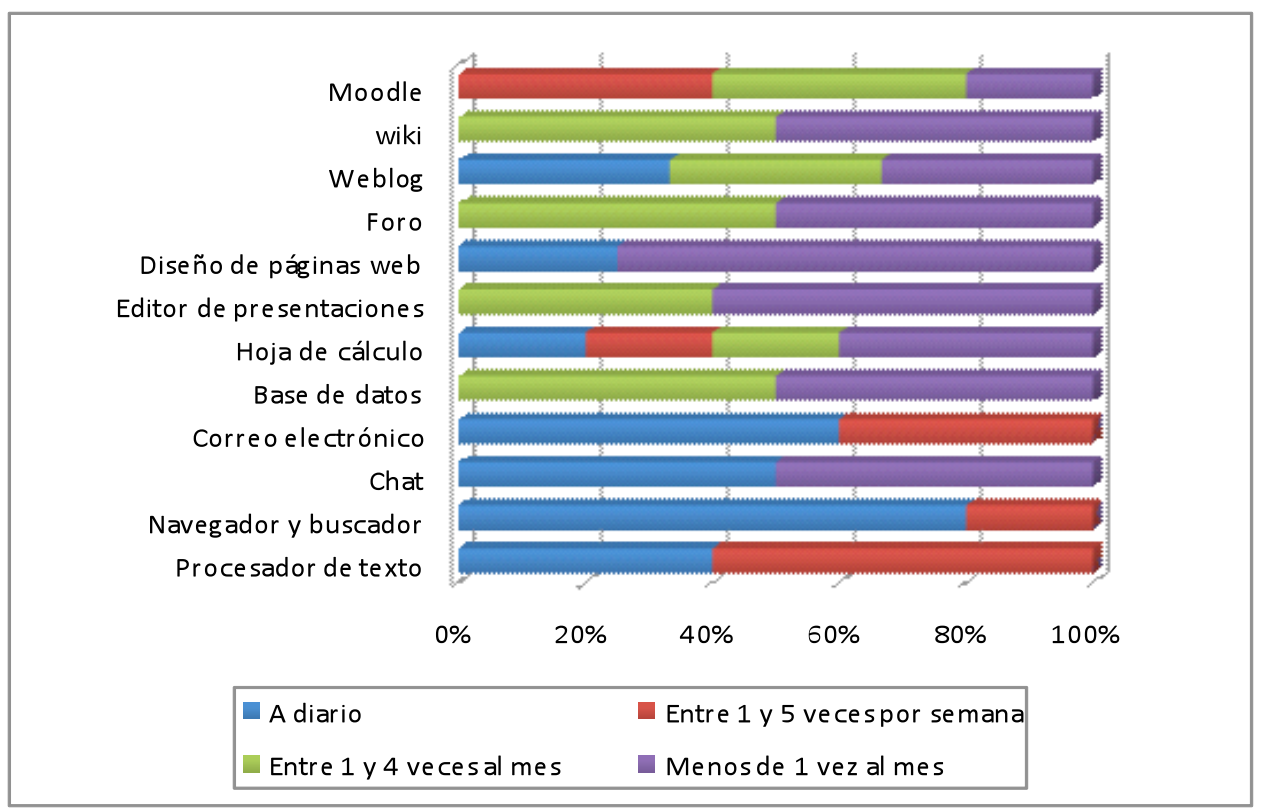

En relación con el esfuerzo que ha supuesto para los profesores el uso de las diferentes herramientas utilizadas para el desarrollo de la experiencia, aquella que ha supuesto un mayor esfuerzo ha sido el diseño de materiales. Ninguno de los profesores ha declarado que ninguna de 
las herramientas haya supuesto nada de esfuerzo, no olvidemos que el aprendizaje de cualquier herramienta supone un esfuerzo por parte del que la aprende, aunque sea poco.

Respecto a las dificultades encontradas por los alumnos para el desarrollo de la experiencia, más de la mitad afirma haber encontrado dificultades diversas a lo largo de la experiencia: con las fracciones, con el ordenador, con el acceso a Internet o con el acceso a Moodle (80\%).

En relación a la posibilidad de trabajar de un modo más autónomo, más de la mitad de los alumnos considera que la experiencia se lo ha permitido bastante o mucho aunque por otra parte menos de la mitad de los alumnos considera que ha aprendido más que en una clase tradicional

La ayuda que los profesores han proporcionado a los alumnos es considerada como buena y excelente por la mayoría de los alumnos, además de que esta mayoría afirma que le gustaría realizar una experiencia similar.

La opinión general de los alumnos acerca de la experiencia de trabajo con objetos de aprendizaje, es bastante positiva ya que casi el total de estos la califica como interesante, fácil, divertida y motivadora.

Por último y en relación con la opinión de los profesores acerca del desarrollo de la experiencia con Objetos de Aprendizaje, todos opinas que las sesiones de formación previas a la experiencia han sido suficientes, siendo las sesiones de Moodle y Lectora las mejores valoradas. Además de lo anterior, casi el total del profesorado considera que Moodle es una herramienta adecuada para alumnos de este nivel.

En cuando a la valoración general que éstos hacen de la experiencia, todos los profesores la han valorado como interesante y motivadora a pesar de que también encontramos un pequeño porcentaje que la considera difícil y aburrida. Con lo anterior, también nos parece interesante comentar la percepción de los profesores acerca de cómo creen que los alumnos han valorado la experiencia ya que aquí encontramos gran variedad en las repuestas ya que estas están en función de la experiencia que cada uno haya tenido en clase con sus alumnos. Más de la mitad considera que la experiencia ha sido interesante, divertida y fácil para los alumnos, mientras que el resto considera que ha sido indiferente, aburrida y difícil. De otra parte más de la mitad de los profesores cree que la experiencia ha resultado desmotivadora, frente al resto que la considera bastante motivadora para los alumnos. Todos los profesores, están de acuerdo en afirmar que la experiencia ha sido bastante satisfactoria aunque opinan que las dificultades técnicas son el aspecto que más a desmotivado tanto a alumnos como profesores y que la madurez de los alumnos para la realización de la experiencia era insuficiente. 


\section{CONCLUSIONES.}

La experiencia de trabajo con Objetos de Aprendizaje y el análisis de los resultados obtenidos durante su desarrollo nos permiten presentar las principales conclusiones sobre la implementación de los objetos de aprendizaje en la enseñanza de las matemáticas en la Educación Secundaria.

\subsection{Sobre los alumnos y los profesores.}

En este apartado atendemos a la dimensión personal del alumno y del profesor haciendo hincapié en la información obtenida sobre las destrezas y capacidades identificadas en éstos durante el proceso de análisis de los datos de nuestra experiencia.

\section{- Los servicios de comunicación de Internet son los más utilizados por los alumnos.}

Los datos han revelado que los alumnos hacen un uso mayor de aplicaciones de Internet, que de herramientas ofimáticas. Resulta destacable que las herramientas que más utilizan a diario sean la mensajería instantánea, seguida de los navegadores y buscadores, mientras que la menos utilizada es el procesador de textos. En las edades de los alumnos que están participando en la experiencia, la mayor parte de los contactos que tienen almacenados en su aplicación de mensajería instantánea son tanto familiares como amigos del colegio o de su entorno social inmediato, por tanto, en el desarrollo de experiencias posteriores puede resultar interesante aprovechar las posibilidades colaborativas de herramientas como la mensajería instantánea (pizarra compartida) y del correo electrónico para favorecer un aprendizaje basado en la construcción compartida del conocimiento.

Al igual que ocurre con los alumnos la aplicación más utilizada por los profesores son los navegadores y buscadores y los servicios de comunicación y colaboración en red como el correo electrónico, el chat y los blogs, lo que vuelve a poner de manifiesto que las herramientas de comunicación y colaboración en red podrían ser utilizadas por los profesores para llevar a cabo situaciones virtuales de enseñanza-aprendizaje, o incluso para apoyar o complementar los procesos desarrollados en la enseñanza presencial para el desarrollo de experiencias posteriores.

\section{- Los alumnos utilizan el ordenador para realizar tareas escolares.}

A pesar de que la mayoría de los alumnos realizan un uso de la red basado en el ocio y en el entretenimiento, casi 9 de cada 10 alumnos participantes en la experiencia señalan que la utilizan para realizar actividades escolares. La mayoría conocen y manejan habitualmente programas de descarga de música y vídeos en red, se comunican por medio de la mensajería instantánea e incluso acceden a juegos en línea, pero también realizan búsquedas en la red relacionadas con las asignaturas que cursan, principalmente de matemáticas, francés y tecnología. 
- Ante las dificultades durante el desarrollo de la experiencia, los alumnos han destacado la predisposición y ayuda brindada por los profesores.

Como en la mayoría de las experiencia de incorporación de las TIC en la enseñanza, los alumnos tuvieron dificultades para el desarrollo de la misma, aunque han destacado como un elemento clave para el desarrollo de la experiencia la predisposición de los profesores a la resolución de problemas y la ayuda brindada por éstos, tanto en el desarrollo de los contenidos conceptuales como en el desarrollo de procedimientos para el manejo de los ordenadores y de Internet. Este puede ser uno de los motivos por el que, a pesar de las dificultades, un 92,2\% de los alumnos han valorado la experiencia como fácil o muy fácil.

\section{- Alto nivel de satisfacción con la experiencia y con los procesos de innovación en el aula.}

En el análisis de estos resultados no debemos perder de vista que se trata de alumnos que se encuentran inmersos en una modalidad de enseñanza presencial, y que por tanto, a pesar del nivel de dominio de las herramientas telemáticas que poseen, no conocen sus funcionalidades educativas y las condiciones de la enseñanza en una situación virtual, por lo que las valoraciones y propuestas de mejora las siguen realizando tomando como referente la enseñanza presencial. Aún así, un 72,6\% de los alumnos estaría dispuesto a continuar o repetir la experiencia, a pesar de que algunos han considerado que aprenden más en una modalidad de enseñanza tradicional $(44,3 \%)$.

\section{- Profesores comprometidos con el proceso de innovación llevado a cabo}

Todos los profesores que se han implicado en el desarrollo de la experiencia poseen un nivel de manejo del ordenador y de Internet adecuado para el diseño de materiales, el apoyo y orientación a los alumnos en la modalidad de enseñanza llevada a cabo, y en las destrezas básicas para aprender a utilizar las herramientas telemáticas seleccionadas para desarrollar la experiencia (Moodle, Lectora y Dspace).

Los datos revelan que la mayoría de los profesores utilizan el ordenador y los servicios y recursos de Internet a diario. De las herramientas ofimáticas más usadas destaca el procesador de texto, sin embargo las hojas de cálculo y las bases de datos siguen siendo las menos utilizadas por los docentes, junto con las presentaciones visuales, a pesar de ser una de las herramientas con más potencialidades didácticas para la enseñanza.

Estas destrezas y competencias mínimas que poseían los profesores ha favorecido que todos ellos hayan considerado la experiencia interesante, y por tanto, que hayan tenido una predisposición muy favorable a desarrollarla con éxito, a aprender las orientaciones básicas para el diseño de materiales didácticos, el diseño de situaciones de enseñanza en red, y la organización y uso de objetos de aprendizaje y repositorios digitales para la enseñanza. También 
hay que destacar que todos ellos se ofrecieron a llevar a cabo esta experiencia por lo que era de esperar una buena predisposición por su parte en el desarrollo de la misma.

\section{- La mayor dificultad experimentada por los docentes ha sido la del diseño de materiales y el manejo de herramientas.}

Sin lugar a dudas, una de las cuestiones que más dificultad ha presentado para los profesores ha sido el diseño de los materiales didácticos para la enseñanza de las matemáticas, ya que ello implicó, en primer lugar, la realización de actividades formativas para aprender a usar las herramientas seleccionadas, y en segundo lugar, el comienzo de la fase de diseño de los materiales, con el consiguiente esfuerzo que ello supuso a nivel de desarrollo de destrezas de manejo de las herramientas (dimensión técnica), de organización de los contenidos (dimensión sémica) y de dominio de los códigos implicados en el diseño de los materiales (dimensión simbólica).

Asimismo, no debemos eludir el esfuerzo que el diseño de materiales y el manejo de las herramientas supuso a nivel temporal y organizativo de los docentes, ya que la realización de estas actividades se tuvieron que llevar a cabo durante el proceso de desarrollo de la investigación, que a su vez coincidió, con el desarrollo de la experiencia en el centro escolar.

A pesar de las dificultades identificadas, los profesores han señalado que las acciones formativas han sido suficientes y la planificación y desarrollo de las mismas adecuado. La selección de las herramientas Moodle y Lectora ha sido considerada como muy adecuada por la mayoría de los profesores, destacando su utilidad y necesidad para el desarrollo de la experiencia, así como la flexibilidad y el carácter organizado de las herramientas.

\section{- Perciben alto grado de satisfacción en el alumnado a pesar de las dificultades técnicas.}

Los profesores han hecho explícito la desmotivación que muchos de ellos, y sobre todo los alumnos, han experimentado por las dificultades técnicas que en algunos momentos se han producido, aunque por otro lado, ellos mismos han comprendido que éstos son inevitables en un sistema de enseñanza apoyado en el uso de las TIC, y que para superarlos es imprescindible un adecuado apoyo técnico, una conexión a Internet que garantice la calidad del servicio, y sobre todo, una planificación didáctica que enfatice los aspectos pedagógicos sobre los técnicos y que plantee alternativas eficaces para evitar la paralización del proceso de aprendizaje iniciado por los alumnos con el uso de las TIC.

Aún así, la mayoría considera que la experiencia ha sido interesante para los alumnos, que su desarrollo no ha implicado un nivel de dificultad elevado para ellos y que se han sentido motivados por participar en ella. Sin embargo, opinan que la experiencia hubiera sido más satisfactoria si se hubiera realizado con cursos superiores, pues el nivel madurativo de los alumnos para responsabilizarse de su aprendizaje y desarrollar una conducta de autonomía era escaso. 


\subsection{Aspectos Pedagógicos.}

En este apartado de las conclusiones, recogemos algunos aspectos referidos al desarrollo de la situación de enseñanza desarrollada con los alumnos en torno a los objetos de aprendizaje diseñados, prestando atención a las condiciones de la modalidad de enseñanza llevada a cabo, el diseño de los materiales, el procedimiento de trabajo de los alumnos, las actividades planificadas y los problemas acontecidos con la evaluación.

\section{- Modalidad de enseñanza: Apoyo a la enseñanza presencial.}

Con la creación del curso de matemáticas en el entorno virtual de enseñanza-aprendizaje Moodle se pretendió crear un espacio en el que los profesores pudieran distribuir materiales didácticos digitales para la enseñanza de las matemáticas. Paralelamente se mantuvieron las clases presenciales, y el acceso al entorno se realizó tanto desde el aula Plumier que se encontraba en el centro como desde los hogares. Así pues, Moodle se utilizó fundamentalmente para el acceso a contenidos (que incluían las actividades), haciendo uso casi exclusivamente del módulo de materiales y no de las herramientas de comunicación.

Atendiendo a los problemas que habían surgido y que habían complicado incluso la realización de las sesiones presenciales según la planificación prevista (por el tiempo empleado en la resolución de dudas sobre el entorno y la resolución de actividades), los profesores llegaron a la conclusión de que sería aconsejable no trabajar de forma paralela los mismos contenidos en clase y en la plataforma, sino que quizás fuese más conveniente separar ambos contextos de trabajo y marcar objetivos diferenciados para cada caso. Matizaron que para el trabajo en red se deberían elegir temas que no presentaran dificultades especiales y que los alumnos pudieran trabajar de modo más autónomo.

Los profesores señalaron que la integración de los objetos digitales en la situación de enseñanza convencional ha supuesto más una dificultad que una ayuda e incluso manifiestan que ha provocado una ralentización de las clases ya que se ha tardado más en trabajar las fracciones que con el método tradicional,

En este caso, se debería contemplar la necesidad de que variaran los objetivos y la organización de la enseñanza, además de los materiales y los contenidos, que en este caso constituían objetos de aprendizaje y fueron la principal modificación incorporada en el proceso de enseñanzaaprendizaje. Asimismo, se debería plantear la pertinencia de utilizar herramientas de comunicación y colaboración en red, y de planificar actividades formativas para docentes orientadas a la planificación de situaciones virtuales de enseñanza-aprendizaje y al manejo de las herramientas para llevarlas a cabo. 


\section{- Diseño de actividades y procedimiento de trabajo de los alumnos}

En un principio la experiencia se planteó de manera obligatoria para todos los alumnos, posteriormente, tras comprobar la realidad del centro, se decidió que se realizará de forma voluntaria y que dicha actividad supondría un incremento en la nota final. Por este hecho nos encontramos que en Moodle, un $30 \%$ de los alumnos no realizaron ninguna actividad. Esto pudo favorecer el aumento de la desmotivación de los alumnos que tuvieran más dificultades para acceder a Internet fuera del centro escolar, o bien tuvieran menos destrezas para el manejo autónomo de las herramientas en red.

Asimismo, los docentes percibieron que el proceso de trabajo de los alumnos variaba mucho con respecto a la realización de la tarea presencialmente. Se percibió que cuando los alumnos estaban delante del ordenador no veían un "ejercicio de matemáticas", y por tanto, no pensaban en el modo tradicional de resolverlo (realizando operaciones en papel). El procedimiento utilizado cuando se enfrentaban con el problema en la pantalla era el de ir probando hasta encontrar la solución, sin emplear métodos reflexivos, críticos y lógicos, es decir métodos y procedimientos matemáticos para su resolución.

El análisis del comportamiento seguido por los alumnos y su manera de proceder ante el problema nos lleva a considerar que el diseño de actividades debería basarse en diseños más interactivos y basados en la resolución de problemas, e incluso podría ser recomendable utilizar metodologías activas basadas en estudios de casos y seminarios de trabajo colaborativos y de tutoría con los alumnos orientados a la discusión y a la reflexión crítica y el análisis de toma de decisiones.

\section{- Problemas en la evaluación de los aprendizajes de los alumnos.}

Los profesores ponen de manifiesto que la realización de las actividades en Moodle ha sido para algunos alumnos frustrante ya que generó mucha inseguridad en ellos que estas actividades fueran evaluadas con un $30 \%$ de la nota final. Para solucionar los problemas generados por la decisión tomada sobre el procedimiento de evaluación, se decidió adoptar un criterio más flexible y utilizar la actividad realizada sólo para subir la calificación tradicional que los alumnos obtuvieran de la realización de los exámenes convencionales realizados a lo largo del trimestre.

Asimismo, se comprobó que hubo problemas técnicos durante la evaluación de las actividades realizadas en Moodle: las evaluaciones que se grababan no siempre respondían a la ejecución real del alumno; cuando trabajaban en el aula Plumier sólo se grababa el resultado del primer alumno, no de los demás que concluían después. 


\subsection{Sobre nuestra experiencia de trabajo con Objetos de Aprendizaje.}

Para finalizar este trabajo, consideramos necesario presentar una serie de conclusiones a las que hemos llegado tras el estudio del tema de Objetos de Aprendizaje y la puesta en práctica de la experiencia con éstos.

\section{- La reutilización de los Objetos de Aprendizaje no siempre es factible.}

Para poder desarrollar nuestra experiencia de trabajo con Objetos de Aprendizaje, los profesores implicados han diseñado los $O A$, ya que se decidió que fueran de un tema concreto: fracciones. Este hecho resulta bastante paradójico ya que la reutilización es una de las principales características de los Objetos de Aprendizaje. En la base del trabajo con Objetos de Aprendizaje, está la idea de facilitar el trabajo de las personas que quieran utilizarlos, ya que simplemente deberían buscar el objeto que necesitan en un repositorio determinado y adaptarlo a sus necesidades concretas, pero la realidad es bastante diferente. Es interesante por tanto potenciar la realización de experiencias en las que el trabajo con Objetos de Aprendizaje se apoye en la reutilización de los mismos, para poder analizar las posibles dificultades y potencialidades de los repositorios, así como su funcionalidad real en la enseñanza.

No olvidemos, con respecto a esta idea, que reutilizar materiales de los repositorios a menudo es una tarea también compleja, pues además de exigir un proceso inicial de búsqueda y selección, exige en una segunda fase una reflexión sobre su integración curricular y además es muy posible que a menudo sea necesario afrontar la tarea de rediseñarlos.

Por todo ello, sería también muy interesante para trabajos futuros analizar el uso que se hace de los repositorios para ver las aplicaciones que se da a los Objetos. $O$ igualmente investigaciones que centradas en contextos reales se planteen el diseño de objetos que estén directamente relacionados con contenidos curriculares.

\section{- Los OA no han muerto.}

A pesar de que Wiley (2006) anunciara la muerte de los Objetos de Aprendizaje, alegando que tal y como éstos estaban planteados con una gran cantidad de requerimientos técnicos e informáticos no eran útiles para la ecuación, son muchas las experiencias que se han llevado a cabo después. El concepto de OA, nació muy unido al mundo de la informática y debido a eso cuando intentamos introducirlos en la educación es difícil que funcionen. Esto no quiere decir que los Objetos de Aprendizaje hayan muerto, sino que se requiere de nosotros un mayor esfuerzo por ves sus potencialidades y limitaciones y así adaptarlos al mundo de la educación.

\section{- Los problemas técnicos no surgen por el uso de Objetos de Aprendizaje.}

Los problemas técnicos que han surgido a lo largo de la experiencia no se refieren concretamente al trabajo con Objetos de Aprendizaje si no que atienden a cuestiones de tipo más amplio como a problemas con las conexiones del centro. El trabajo con los Objetos de Aprendizaje, en concreto, 
no ha presentado problemas técnicos importantes que hagan que los profesores desistan de su uso en posibles ocasiones futuras. Esta innovación en torno al diseño de materiales es sencilla de implementar al contrario de lo que puede ocurrir con otro tipo de experiencias. Se hace necesario por tanto aprovechar estas ocasiones en las que la introducción de las TIC en el aula es fácilmente realizable para sensibilizar a los profesores y hacerles ver que es posible trabajar con las TIC, ya que en la mayoría de las ocasiones nos acercamos a la tecnología con miedo, incertidumbre y un gran escepticismo que hace que no aprovechemos todas sus posibilidades. (Martínez y Prendes, 2001, 2003).

\section{- Sobre las comunidades de profesores.}

La experiencia que hemos realizado con los Objetos de Aprendizaje, ha sido llevada a cabo por una comunidad de profesores bastante pequeña. En estas situaciones, el trabajo es mayor para cada uno de los profesores y más si contamos con el hecho de que hay que partir de cero y diseñar y producir los objetos. Por el contrario, cuando se trabaja con una comunidad de profesores relativamente mayor, por ejemplo todos los profesores de un IES, o todos los profesores de matemáticas de una ciudad, el trabajo es menor y es posible reutilizar los objetos diseñados por otros. Trabajando con más profesores, los resultados y ventajas del uso de los OA, son mayores y a corto plazo, ya que el esfuerzo de producir un material determinado se ve compensado por la posibilidad de utilizar otras materiales producidos por otros profesores.

\section{Bibliografía.}

- CABERO, J. Y OTROS (1999): Tecnología educativa. Síntesis. Madrid.

- GURELL, S.(2008): Open Educational Resources Handbook for Educators 1.0. Center for Open and Sustanaible Learning

- L'ALLIER, J. (1997) Frame of Reference: "NETg's Map to the products. Their Structure and Core Beliefs". En http://www.netg.com/research/whitepapers/frameref.as (Consultado el 10 de Mayo de 2008).

- MARTÍNEZ SÁNCHEZ, F. Y PRENDES ESPINOSA, M.P. (2001): "La innovación tecnológica en el sistema escolar y el rol del profesor como elemento clave del cambio". Educar en el 2000. Revista de Formación del Profesorado. (3), 14-17.

- MARTÍNEZ SÁNCHEZ, F. Y PRENDES ESPINOSA, M.P. (coords.): "La enseñanza con objetos de aprendizaje". Madrid: Dykinson. 2008

- MARTÍNEZ, F. y PRENDES, M. (2003) “Redes para la formación” En MARTínEZ, F. Redes de Comunicación en la enseñanza. Barcelona: Paidós.

- MARTínez, F. y PRENDES, M.P. (2003) “¿Adónde va la educación en un mundo de tecnologías?". En MARTíNEZ, F. (2003) Redes de comunicación en la enseñanza. Barcelona: Paidós

- MUIRHEAD, B. \& HAUGHEY, M. (2003): An assessment of the learning objects, models and frameworks developed by the Learning Federation Schools Online Curriculum Content 
Initiative. Documento en línea (consultado el 10 de Mayo de 2008) en: http://www.thelearningfederation.edu.au/tlf/newcms/view_page.asp?page id=8620\&M en $I d=4$

- NATIONAL LEARNING INFRASTRUCTURE INITIATIVE (NLII) (2003): Learning objects (NLII 2003). Documento en línea (consultado el 15 de Marzo de 2008): http://www.educause.edu/nlii/keythemes/LearningObjects.asp

- PRENDES, Ma P (1998) Proyecto Docente en Tecnología Educativa. Documento Inédito.

- PRENDES, Mạ P. (1995). “Redes de cable y enseñanza”. En J, Cabero,y F. Martínez Sánchez. Nuevo canales de comunicación en la enseñanza. Madrid: Centro de Estudios Ramón Areces.

- REBOLLO, M. (2004): El estándar SCORM para Ead. Tesina del Máster en Enseñanza y Aprendizaje Abiertos y a Distancia: Universidad Nacional de Educación.

- RELAN, A. \& GILLIANI, B. (1997) Web-based instruction and the traditional classroom: Similarities and Differences. In B.H. Khan (Ed.). Web-Based Instruction. Englewood Cliffs. NJ: Educational Technology Publications.

- VARAS, L.M. (2003): "Repositorio de Objetos de Aprendizaje". http://www.alejandria.cl/recursos/documentos/documento varas.doc (Consultado el 15 de abril de 2008).

- WILEY, D. (2000): “Connecting learning objects to instructional design theory: A definition, a metaphor, and a taxonomy". En D. A. Wiley (Ed.): The Instructional Use of Learning Objects: Online Version. Retrieved MONTH DAY, YEAR, en World Wide Web: http://reusability.org/read/chapters/wiley.doc

- WILEY, D. (2006): "RIP-ing on learning objects". Blog publicado el 9 de enero de 2006 en http://opencontent.org/blog/archives/230 (Consultado el 15 de Marzo de 2008).

- WILEY, D. (2008): “Pasado, presente y futuro de los Objetos de Aprendizaje”. Conferencia plenaria del XI Congreso Internacional Edutec 2008: Puente entre culturas; Iberoamérica y Europa. Documento Inédito.

\section{Para citar este artículo:}

GUTIÉRREZ, Isabel (2008) «Usando objetos de aprendizaje en enseñanza secundaria obligatoria» [artículo en línea]. EDUTEC, Revista Electrónica de Tecnología Educativa. Núm. 27/ Noviembre 2008. [Fecha de consulta: $\mathrm{dd} / \mathrm{mm} / \mathrm{aa}$ ].

http://edutec.rediris.es/revelec2/revelec27/

ISSN 1135-9250. 